\title{
Virtual Tutor: A System for Deploying Digital Libraries in Classrooms
}

\author{
Joemon M. Jose, Hywel Braddick, Innes Martin, Brian Robertson, \\ Craig Walker, and Greg MacPherson \\ Department of Computing Science \\ University of Glasgow, Glasgow G12 8QQ, Scotland, UK. \\ $\{j j$, braddihd, martini, robertsb, walkercp, macphegf $\} @ d c s . g l a . a c . u k$
}

\begin{abstract}
In this paper, we introduce a collaborative learning environment called Virtual Tutor for exploiting valuable digital libraries for teaching and learning. Virtual Tutor facilitates the building of online lectures and their provision. It also supports collaborative and explorative learning. We describe the architecture of the system and its functionality. A summative evaluation is conducted on the system. The paper concludes by comparing the Virtual Tutor functionality with similar approaches and highlighting the strengths of the Virtual Tutor design.
\end{abstract}

\section{Introduction}

The proliferation of powerful computing systems and network technologies have greatly increased the ease with which digital resources are created and shared in both commercial and educational organisations. This increase, introduces the possibility of open-ended teaching and learning activities. Realising this potential, a number of educational digital resources have been created under the banner of digital library initiatives (e.g., JISC (www.jisc.ac.uk), NSF (www.nsf.gov) digital libraries activities). In addition, the Internet hosts valuable digital information that can also be used for teaching and explorative learning. However, our developing prowess in casting data in electronic form is not matched by any compatible facility for effective usage.

Online resources together with emerging network technologies provide new opportunities that were not feasible in traditional libraries for learning activities. In addition to the obvious way of delivering teaching materials online, it opens up the possibility of collaborative and explorative learning. Teachers can use digital resources to find relevant materials on the topic of discussion. This is faster than traditional paper and book based mechanisms and will lead to more timely and up-to-date information. The selected digital documents can then be provided to students as supporting material. Students will be able to use these resources in the context of discussion, and in addition, will also be able to use the digital resources for enhancing their knowledge. There exists a potential for explorative learning and online collaboration. However, these possibilities are under utilised due to the lack of proper tools. 
In this paper, we introduce a collaborative learning environment called Virtual Tutor. This paper is organised as follows. In Section 2, we discuss issues related to online learning and derives a set of criteria for our development. Section 3 introduces the Virtual Tutor design and the rationale behind this design. In Section 4, we discuss a summative evaluation. We argue the power of our design in Section 5 and conclude in Section 6.

\section{Background}

\subsection{Online Digital Resources}

Solvberg [7] defines a digital library as one consisting of digital collections, a working environment and associated technologies and services. A collection contains information objects put together according to some rules or ideas. In order to support users in the information seeking process, catalogues, indexes and retrieval services are provided. For example, JISC in UK supported the creation of a number of digital libraries for online learning (e.g., AXIS (http://www.axisartists.org.uk), EEVL (http://www.eevl.ac.uk)). In addition, there exists repository like the World Wide Web where information is deposited without adhering to any specific rules.

These digital resources are valuable knowledge sources for teaching and learning purposes. Many academics place the latest essays and research articles on the Web, making it an important place for up-to-date information. A given domain may be supported by a number of specialised digital libraries. In order to use these resources for learning \& teaching, one has to develop techniques to find and use the information in teaching. In addition, these materials need to be delivered online for easy and flexible access. Finding relevant information from a number of sources using search engines with different technologies is a difficult problem. Once relevant sets are retrieved, identifying and using a set of useful documents is another problem. In this work, we address these issues by building a workspace in which different search engines can be represented. It also provides facilities to view and compare documents for selection purposes [4].

\subsection{Online Teaching and Learning}

Online delivery of course materials can be extremely flexible. This flexibility comes from two angles. Firstly, students can explore the material at their own pace and time, and is a viable substitute for face-to-face learning. Secondly, it provides an opportunity to teaching staff to constantly rectify and revise their materials based on student feedback. A re-examination of the objectives and the organisation of course materials can lead to improvements in the way that topic is taught. This makes the materials of superior quality and up-to-date.

However, in order to make online learning successful, it should engage the student in the learning material. Milligan [6] argues that merely using 
electronic delivery as a means of enhancing the presentation of learning material is not enough. This is because, Milligan continues, the face-to-face learner gets far more from a lecture than merely the information that is written on the blackboard or spoken by the lecturer. In addition to this formal learning, they get the chance to meet and discuss issues with their peers, they have the opportunity to interrupt their lecturer when they fail to understand, they get an indication as to how quickly they should be progressing through the materials etc. This type of interaction helps to reinforce learning, and catch misconceptions early. In order to make online delivery effective, all these 'extras' have to be delivered alongside any online learning material.

Our goal is to build a system that supports active exploration, and learning. This system should keep the flexibility of online delivery while providing the benefits of classroom teaching. Basically, an ideal online learning material should extend beyond being a virtual course book, to being a virtual classroom.

\subsection{Objectives}

In this work, we aim to develop a collaborative environment for explorative learning. Our idea is to support collaboration through peer-to-peer consultation and to support exploration through the use of multiple digital resources. People learn more effectively by talking to others and swapping information and ideas. The basis of our approach is the belief that good online learning environment must provide not just the knowledge or information, but also the opportunity for communication and reinforcement of learning through reflection and collaboration. In the following we describe the desiderata for our work.

\section{Desiderata}

- Support Lecture preparation: While preparing course notes, Lecturers always look for additional material (on multiple digital resources) to verify a fact and/or to provide additional relevant material to students.

- Support explorative learning and collaboration: It is important to facilitate explorative learning. That is, students should be able to probe related materials (on multiple sources) and share their ideas with peers thus supporting collaboration.

- Support Flexibility: A learning environment should ideally offer the same flexibility as a piece of paper. Students take notes when they listen to a lecture and hence the system should provide a comparable facility.

\section{Our Approach}

To meet the criteria described above, we designed and implemented a system called Virtual Tutor. A sketch of the interface is shown in figure1, Virtual Tutor can be used to create lectures. Lecture materials can be presented to the students 
with associated and supporting information. Teachers will be able to create a series of slides with links to relevant resources. Students will then be able to view the prepared lectures, adding their own comments, questions and links if they so desire. Since students can see what other students have taken from the lecture, they can use this information to make the best use of their time whilst using the facility. This will also provide feedback for lecturers on the material provided and highlight any areas of difficulty.

\section{$3.1 \quad$ Interface}

The layout of the main interface is critical to the success of creating a useful learning environment. The interface consists of two main parts, an overview window and the workspace. The workspace is designed to be a flexible area used to aid problem solving and organisation. The overview window will contain several different views of the overall structure of the current lecture (see figure 2). Both horizontal and vertical scrollbars will be used in order to give the impression of infinite size although, in reality, this size will be finite. The size of each component will be inversely proportional to the other with the use of a dynamic horizontal split pane, thus enabling the user to customise the main display depending on the task in hand. The following description gives the rationale behind the design decisions regarding each component included within this interface.

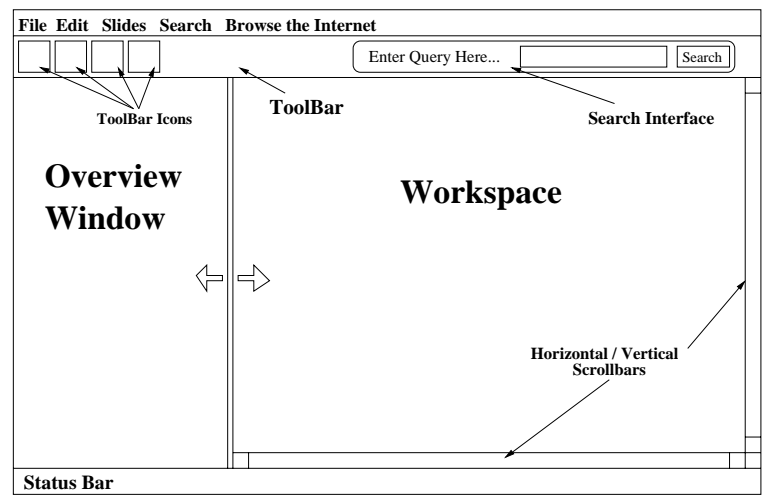

Fig. 1. Virtual Tutor Interface Design

\subsection{Rationale behind This Design}

The overview window will provide a hierarchical organisation of the slides and other associated information such as links to supporting materials, questions presented by the students and their answers (figure 21). It basically provides 
a structured view of the material. Comments, links, and questions are represented with distinguishable icons for easy understanding. Lecturers can use the 'miniSlides View' to create slides. The idea behind the workspace is to provide flexibility equivalent to a piece of paper.

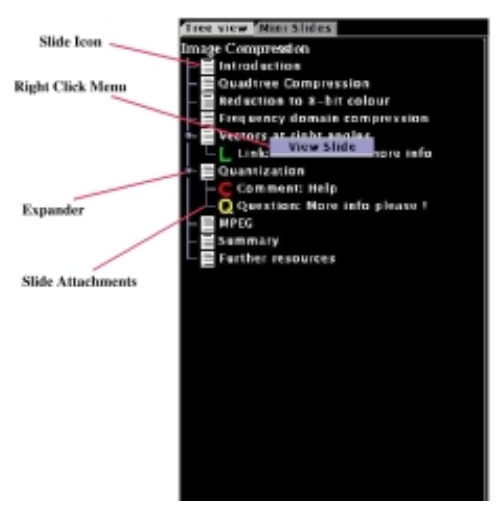

Fig. 2. Slide Viewer

People always need additional resources when teaching and learning. It is important to help students/staff to explore additional materials to clarify an issue or aspect. Lecturers always look for supporting material for emphasising a point. Partly due to the growth of the Internet, both in popularity and quantity of online data, it is increasingly common for academics to use the Internet as their first, and in many cases, only reference. In specialised environments, a number of digital resources may be available for a given domain. Examples are the digital collections from the JISC. Search functionality needs to support finding information from these resources. This will allow lecturers to look for information to construct a lecture, look for examples and counter examples. In addition, students may use these clues and explore this collection to look for additional materials (for example to write an essay). In the Virtual Tutor one can represent multiple search engines. Currently, we provide search facility for the Internet. A user can issue a search to a single search engine or more. The results will be displayed along with query-biased document summaries [89. The workspace will allow selecting and viewing these retrieved set of documents. Users can drag and drop selected items to the workspace and link to the slides if needed. In addition, it allows them to group materials and store them for later use.

An important issue is to develop a medium for students to share their interpretations of a piece of information with each other and to explore the connections between this item and outside materials, including imagery, sounds, texts, and personal experience. This enables students to record their work, share 
it with others, and view and comment upon the work of others. The workspace acts as a medium for recording learning activities similar to taking notes on paper. Levy \& Marshall [5] have shown that individuals "generally do not take notes by writing their observations down on a separate sheet of paper or in a text editor... Instead, they mark on the documents themselves". For example, students often add annotations during lectures by marking the original printed lecture notes. These annotations (i.e., questions, links, comments, answers) would certainly be useful to the annotators themselves, but the sharing of annotations is an important way in which we use documents to collaborate. For example, students would have access to the annotations of fellow students, which would introduce all the benefits of collaborative learning as discussed earlier.

The workspace facilitates this activity by allowing students to note down questions and comments, and linking them to slides or slide groupings. In fact, this feature leaves traces of user actions behind, thus providing an opportunity to follow-it-up later. A system has been implemented in Java programming language and uses XML for data interchange between components. A snapshot of the interface is shown in figure 3.

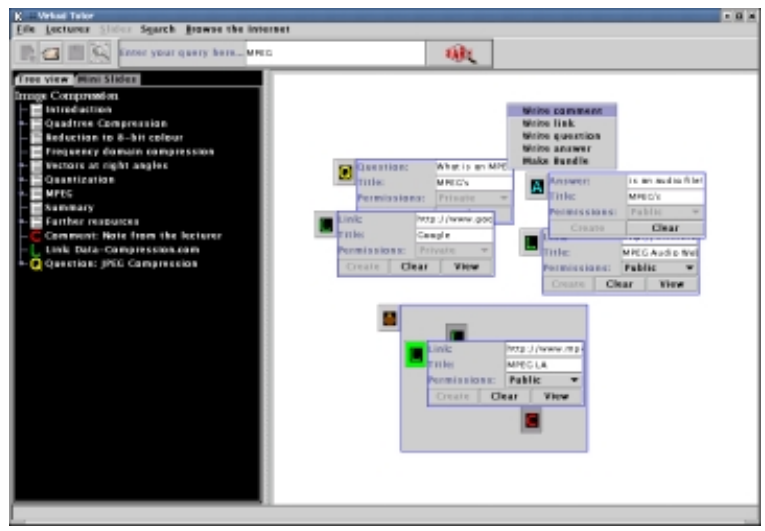

Fig. 3. Virtual Tutor Interface

\subsection{Superimposition of Information}

In the above section, we described the rationale behind our design. The Virtual Tutor can represent material such as a link to a Web resource, questions from students on a slide or a section, answers from lecturers, and comments. These, in a way, are a super imposition of information [1] on the basic lecture material. 
In order to provide a flexible interface, we identified a hierarchy of superimposed information as described below:

- link - A link represents a shortcut to some resource. In general, resources may be anything such as video clips or databases but for the purpose of our prototype, the links will only provide shortcuts to web pages.

- Comment - If a user has something to add or say about a lecture, such as recommending a link or adding some extra information to expand on a slide then they can create a comment object and attach this to the lecture where appropriate.

- Question - If the user has a specific question about a slide, group of slides or the entire lecture, they can create a question object and attach it to the relevant part of the lecture.

- Answer - When a user sees a question which they are able to answer, they can create an answer and attach it to that question. Questions will not be limited to only having one answer so that multiple perspectives / viewpoints for each question can be seen.

- Bundle [2] - It will often be the case that users will want to add several of the above components to the lecture which are all related. Instead of adding these objects individually the user will be able to create a single bundle object with any number of objects inside. The bundle can then be added to the lecture where appropriate.

The class hierarchy shown in figure 4 describe various information units and their relationships. Similar objects will be made to inherit from the same superclass and hence share common properties, so can be treated in a generic manner where suitable. The main class will be the Lecture class which will store references to all components in the lecture as well as reference tables which will store the relationships between the components. The class diagram in figure 4 highlights the inheritance and dependency relationships between the classes.

\subsection{System Features}

When users create superimpositions they will be able to decide whether others will be able to view that component. This allows users to have private information in their view of the lecture whilst still enabling collaboration as the user can make components viewable to everyone by declaring them as public. The only user who will have permission to see all superimpositions, whether public or private, is the author of the lecture. This will allow the lecturer to see what problems people are having and to answer any questions which students may have made private.

A user can have his own work-space on which he can create and manipulate objects either to be included in the lecture or for general problem solving. This provides the user with an area of temporary storage where he can leave objects which may only be used for that session and not attached to the lecture. The 


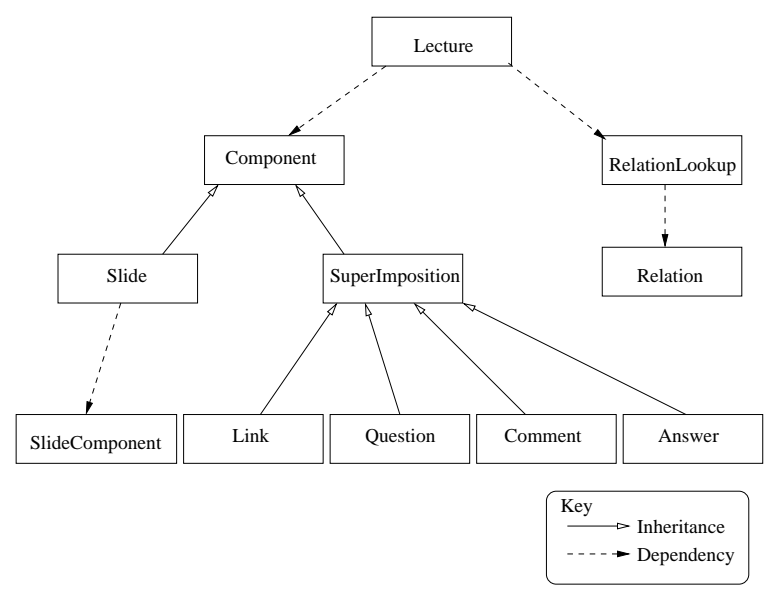

Fig. 4. Data Model class diagram

user will be able to add several objects to the workspace and then select these objects to be collectively added to a bundle. The workspace will also allow users to arrange their own personal information in a way that best suits them, thus giving a high degree of customisation and flexibility.

There are two main views for the lecture. One of these views is to be used to display an overview of all the slides, also indicating if there are any other components attached to each one. The other view is to be a structured hierarchical view showing all the components of the lecture and the relationships between them (see figure 2). If a user wishes to view an individual slide, he can select it from either of the viewers to be displayed in an individual slide viewer. The user will be able to have several slides open in his own viewers at the same time, thus allowing him to view and compare several different slides simultaneously.

When the user wishes to carry out a search, he will be able to select the domains he wishes to submit the query to. There will be a separate set of results returned for each of the domains selected (figure 51). Once the results have been returned, the user will then be able to view the results. If the user wishes, he will then be able to drag the link from the results to the workspace or directly on to the lecture. In order to help the user to judge the relevancy of a retrieved document, we have incorporated query-biased summaries as well [89].

\section{Evaluation}

We conducted a summative evaluation to investigate the strengths and weaknesses of the Virtual Tutor design. We used the following instruments for con- 


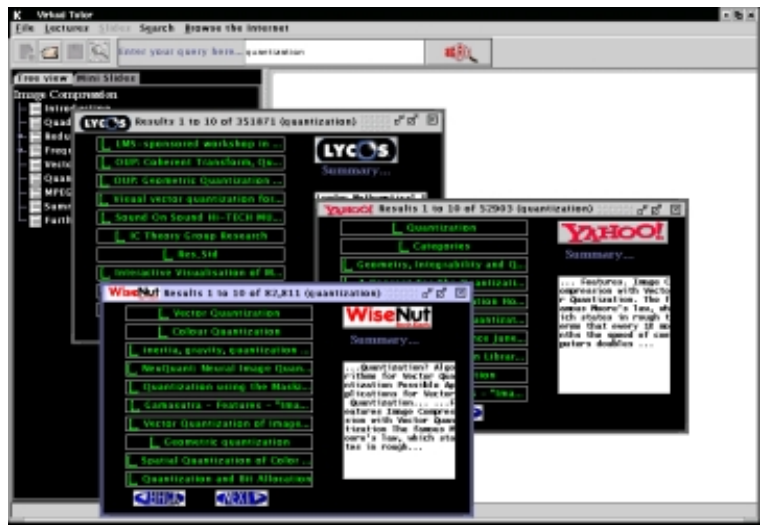

Fig. 5. Search facility in virtual Tutor

ducting the evaluation: Think aloud observation; Interview; and Questionnaire. Subjects were given a quick reference guide and basic introductory session. They were expected to be able to use the basic functions of the program after having read this guide. The subjects were then given a number of tasks to complete, and were expected to try and solve these tasks without any further help from the evaluator. It was hoped not only that the techniques would allow specific problems in the design to be identified, but also to assess the system's functionality as well as usability. This includes the efficiency, effectiveness and satisfaction.

We used twelve undergraduate students. Four of them were chosen to go through the think aloud observation. The other eight users were either interviewed or filled out a questionnaire. From the questionnaire, it was gathered that most subjects were complimentary about the layout and system functionality. Think-aloud was definitely the most informative of the three evaluation techniques used, as is often the case when evaluating a graphical user interface. It gave valuable insight into how users interact with the system, and reveal their strategies for overcoming any problems encountered. Subtle user behaviour such as expecting a link to pop up from the tree view after a double-click was also discovered, as was the need for clearer feedback from the system after user actions. From the interviews conducted, it appeared that although most of the users were happy with the system, some minor interactions such as viewing super impositions (comments, links etc) could be improved. Users felt that the layout was adequate, the bundling technique was fine and the lecture material was presented clearly. From the three evaluation techniques carried out and through our own internal evaluation, it is clear that a solution has been developed that can be seen as a success. The attempted solution to the organisation of large amounts of heterogeneous data through our bundling technique has proved to be quite successful. 


\section{Discussion}

Despite evident usefulness, collaboration with a view to learning is one area in which the paper medium still seems to be preferred to the digital. How does Virtual Tutor make progress on this issue? What are the strengths and weaknesses of the Virtual Tutor, and how it does contribute to online learning? In this section, we try to answer these questions and compare Virtual Tutor against other online learning environments.

Most of the research to-date addresses the issue of providing material online and supporting this process by developing administrative tools [6]. These systems are generally known as MLEs (Managed Learning Environments), VLEs (Virtual Learning Environments) etc. The main issues addressed are administrative and those of basic technology. These approaches overlook the ability of networks to bring people together in new configurations to support open-ended learning activities. We believe the Virtual Tutor design is a quantum leap forward compared to these systems.

Virtual Tutor facilitates collaboration by allowing students to ask questions and record them to the particular point of contention. The workspace facility allows them to record each action in such a away that others can get benefit out of them. We believe, as Hendry 4 argued, that leaving traces of their previous actions will facilitate collaboration. Possibly the most important concept that introduced in the development of this prototype has been the organisation of heterogeneous data into easily understandable, higher level groupings, where the user can group slides along with comments, links and questions to be added to lectures or left on the shared workspace for others to view. In addition to these, the workspace allows the representation of many different search engines and is extensible. Indeed, we have represented search facility from many different web search engines (figure 5). The results are presented with a query-biased summary of each retrieved document, thus facilitating easy relevance judgement 89 . This is one of the important features that makes the Virtual Tutor design different in comparison to other tools such as CSILE (http://csile.oise.utoronto.ca/intro.html).

Wilensky 10 defines spontaneous collaboration as "the ability of users to collaborate without first undertaking a substantial mutual administration commitment". Wilensky continues that this concept is likely to have a profound effect on the process by which lecturers/students teach and learn with the possibility of superseding traditional paper-based lecture notes or even the entire concept of lectures as we know them today. The workspace in the Virtual Tutor is designed with a view to enhance collaboration. On the workspace, one can take notes, make annotations and mark questions as one does with paper-based lecture notes. In addition, one can search for additional materials relevant to a topic and incorporate them into the workspace. This in a way leaves traces of one's activity and is helpful to that individual as well as others in understanding a topic of discussion. 
In such a collaborative environment, people can work together across time and space outside normal class time. We believe that Virtual Tutor enables learners to accomplish tasks and develop understanding beyond what they can achieve individually, by making use of distributed expertise and multiple perspectives. In addition, as argued in [3], the communication that is required to support collaboration forces learners to articulate their understanding in ways that help them to organise their knowledge and acknowledge gaps in their understanding. We believe the Virtual Tutor makes an important contribution towards this end.

Recent educational research focuses on the value of collaboration and of openended activity for learning. The Collaboratory Notebook Project at Northwestern University investigates issues related to collaboration and open-ended learning [3. The Notebook was designed specifically to support communication and collaboration among students, instructors, and mentors, and to allow people to work together across time and space outside the normal class time. However, the notebook is rigidly structured and quite over-determined which limits the flexibility of the application. The similarity between the Notebook and the Virtual Tutor is that of slide overview. The Notebook organises the system similar to that of slide overview (figure 22). Compared to this, the Virtual Tutor interface provides much more flexibility by the introduction of a workspace. In addition, Virtual Tutor provides extensible search facility because one of our aims is to make use of digital resources for explorative learning, whereas Notebook concentrates on the online delivery of teaching materials.

\section{Conclusion \& Future Work}

In this paper, we have introduced a collaborative learning environment called Virtual Tutor for exploiting digital libraries for explorative learning. Virtual Tutor can be used to create lecture materials. For this, a lecturer can search multiple collections and select relevant information which can be presented to students within the context of individual slides. Lecturers will be allowed to build up a series of slides with links to relevant materials. Students will then be able to view the prepared lectures, adding their own comments, questions and links if they so desire. Since students can see what others have taken from the lecture, they can use this information to make the best use of their time whilst using the facility. This will also provide feedback for lecturers on the material provided, and highlight any areas of difficulty which can then be addressed.

Our aim is to use digital libraries for explorative learning. We have been successful in building a prototype and the summative evaluation showed its usefulness. The system allows us to represent multiple search engines and provides techniques to select and use the retrieved set of results. In future work, we would like to investigate how to use ever increasingly available video and image materials in the system. In addition, we are also planning an evaluation in an operational setting. 
Acknowledgements. Our thanks to Ryen White for providing the WebDocSum search system and assisting with its installation.

\section{References}

1. Bowers, S. A generic approach for representing model-based superimposed information. Research proficiency exam report, Oregon Graduate Institute, May 1, 2000 .

2. Delcambre, L. M. L., Maier, D., Bowers, S., Weaver, M., Deng, L., Gorman, P., Ash, J., Lavelle, M., And Lyman, J. Bundles in captivity: An application of superimposed information. In Proceedings of the 17th International Conference on Data Engineering (ICDE 2001) (April 2-6 2001), IEEE Computer Society Press, pp. 111-120.

3. Edelson, Daniel, C., Pea, Roy, D., And Gomez, Louis, M. The collaboratory notebook. Communications of the ACM 39, 4 (1996), 32-34.

4. Hendry, D. G. Extensible Information-Seeking Environments. PhD thesis, The Robert Gordon University, Aberdeen, September 1996.

5. Levy, D. M., And Marshall, C. C. Going digital: A look at assumptions underlying digital libraries. Communications of the ACM 38, 4 (April 1995), 77-84.

6. Milligan, C. Virtual learning environments in the online delivery of staff development. JTAP report, Heriot-Watt University, URL: http://jisc.ac.uk/jtap/htm/jtap-044.html, 1999.

7. Solvberg, I. T. Digital libraries and information retrieval. In LNCS 1980 (Proceedings of ESSIR 2000) (2000), M. Agosti, F. Crestani, and G. Pasi, Eds., Springer-Verlag, Berlin, Heidelberg, pp. 139-156.

8. White, R., Jose, J. M., And Ruthven, I. Query-biased web page summarisation: A task-oriented evaluation. In Proceedings of the Twenty Fourth Annual International SIGIR Conference on Research and Development in Information Retrieval (September 2001), ACM Press, pp. 412-413.

9. White, R., Ruthven, I., And Jose, J. M. Web document summarisation: a task-oriented evaluation. In Proceedings of the First International Workshop on Digital Libraries DLib2001 (September 2001), IEE Press, pp. 951-955.

10. Wilesky, R. Digital library resources as a basis for collaborative work. Journal of the Americal society for Information science 51, 3 (2000), 223-245. 